Egyptian

Orthodontic Journal

\title{
DENTAL AND SKELETAL CHANGES PRODUCED BY RAPID MAXILLARY EXPANSION (RME)
}

\author{
"IBRAHIM AL-SHORMAN. \\ *JUMANA TBIESHAT. \\ ${ }^{\star * *}$ TALAT MNEIZEL.
}

\section{ABSTRACT}

The primary purpose of this study is to evaluate the dental and skeletal (orthopaedic) affects on the maxilla and its surrounding structures in response to rapid maxillary expansion treatment involving the use of active banded maxillary expansion appliance.

This study involved two experimental groups, each consisted of 6 patients, aged 12 to 15 years wearing hyrax appliance (hygienic rapid maxillary basal arch expansion appliance).

The rapid expansion period lasted 2-4 weeks, followed by a retention period of 12 weeks, followed by post-retention observations for 12 weeks.

The results showed that (RME) appliances were capable of producing significant increases in intermolar width, palatal widt $\hat{h}$, alveolar tipping, and molar tipping and were capable of expanding the maxilla.

\section{INTRODUCTION}

(RME) has been performed for many years to correct skeletal and dental transverse discrepancies between the maxilla and the mandible through a combination of skeletal expansion (separation of the maxillary mid-palatal suture) and dental expansion (lateral tipping of maxillary posterior teeth).

\footnotetext{
* Major

** First Lieutenant

*** Colonel
} 
(RME) is unique among therapies, since the bony movements ramify beyond the dento-alveolar complex; Hass (1965) found that in patients treated with (RME), the maxilla was displaced downward and forward . Similarly, Wertz (1970) noted that the maxilla has moved downward 1 to $2 \mathrm{~mm}$ on a regular basis. Multiple daily activations could result in cumulative loads of 20 pounds or more (Issacson et al.,1964).

The maxilla could then be expanded at a rate of 0.2 to $0.5 \mathrm{~mm}$ per day by opening the mid-palatal suture.

This concept means that high magnitude forces overcome the skeletal resistance with minimal tooth movement within the alveolar process. In addition, (RME) has also been found to have fundamental effects on respiration (Grey LP, 1987).

\section{Subjects and methods}

The experimental sample consisted of 12 patients, 6 boys and 6 girls presenting either a bilateral or a functional unilateral cross bite, they were divided into two experimental groups:

Group one: consisted of 6 patients aged 12 years and 6 months to 14 years and 8 months, treated in King Hussein Medical Centre (KHMC).

Group two: involved 6 patients aged 11 years and 6 months to 15 years, and were treated in Princess Haya AL Hussein hospital.

Since (RME) imposed a short time scale of up to only one month, the effects of growth could then be disregarded and a control group therefore was unnecessary.

All patients needed posterior transverse expansion and all were treated with banded (RME) appliance. The arms of the expansion screw were soldered to the bands that were fitted on the first premolar and first permenant molar as shown in Fig. 1 and 2

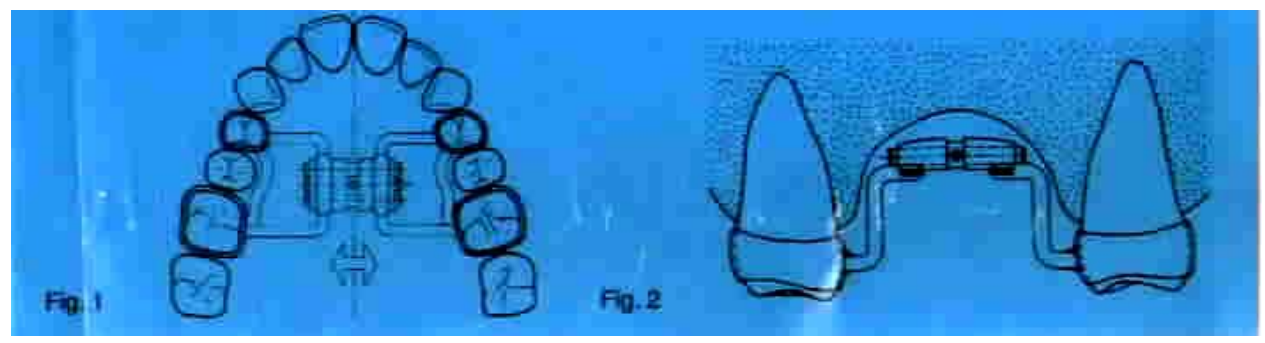

Fig.1 shows the design of (RME), an occlusal view.
Fig.2 shows (RME) in the transverse plane at the level of upper first molar. 
Both groups had similar treatment parameters and were retained for stabilization for approximately 12 weeks.

\section{Models}

Maxillary impressions were taken prior to treatment, at the end of expansion, at the end of retention and at the end of the observation period

The models were trimmed with the base parallel to the occlusal plane, and were evaluated via palatal contour tracings and photographs for changes in intermolar width, palatal width, palatal depth, alveolar tipping and molar rotation.

The inter-canine and inter-molar distances were recorded twice to the nearest $0.1 \mathrm{~mm}$ with dental dial caliper as shown in Fig.3

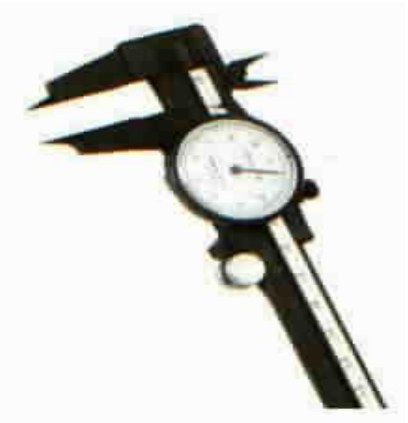

Fig.3

\section{$\operatorname{Radiographs}(\mathbf{R} / \mathbf{G s})$}

- Occlusal (R/Gs) were taken before and 2 weeks following insertion and activation of the appliances and were evaluated for evidence of separation of the mid-palatal suture .

- Lateral cephalograms were traced and analyzed for all patients by customized computerized cephalometry.

The skeletal, dental linear and angular changes during the active phase of (RME), the retention and post-retention period were documented . recorded:

Changes in the following measures before and after treatment were 
a) MPA ( Madibular Plane Angle ) and PPA ( Palatal Plane Angle ) .

b) SN-MP ( Sella Nasion - Mandibular Plane ) and SN-PP ( Sella Nasion Palatal Plane ).

c) Na-Me ( Nasion- Menton)

d) Na-UIE ( Nasion -Upper Incisal edge ), upper anterior facial height (AFH) .

e) DCT-SN ( Maxillary Molar Distal cusp tip - Sella Nasion ) Upper posterior facial height $(\mathrm{PFH})$.

\section{RESULTS}

\section{Clinical findings}

During the expansion period $(0.2-0.5 \mathrm{~mm} /$ day $)$ for $1-3$ weeks , all patients developed midline diastema.

The periodontal condition of the maxillary teeth examined 12 weeks following retention ,appeared excellent in the two groups of our study.

Dislodgement and breakage of the appliance in one patient of group one has occurred.

There was a slight compensatory buccal uprighting of the mandibular posterior teeth, resulted from expansion of the maxillary arch , and the resultant occlusal force.

\section{Cast Analysis}

Table 1: describes the expansion gained between the canines and the molars , and the total relapse calculated during the retention (12 weeks) and postretention period (12 weeks) on casts of group 1 and $2(\mathrm{~mm})$.

\begin{tabular}{|c|c|c|c|c|c|}
\hline & \multirow{2}{*}{ Age } & \multicolumn{2}{|c|}{ Intercanine } & \multicolumn{2}{c|}{ Intermolar } \\
\cline { 3 - 6 } & & Expansion & Relapse & Expansion & Relapse \\
\hline Group 1 X & 13.7 & 8.4 & $2.3(27 \%)$ & 10.6 & $2.8(26 \%)$ \\
\hline Group 1 (s.d) & & 1.1 & 0.5 & 1.5 & 0.8 \\
\hline Group 2 X & 13.3 & 8.8 & $2.6(29 \%)$ & 9.8 & $2.5(25 \%)$ \\
\hline Group 2 (s.d) & & 1.9 & 0.3 & 1.1 & 0.6 \\
\hline
\end{tabular}

Table 1 
In all patients of the two groups, the intermolar distance increased more the intercanine distance, the increase in intermolar width produced linear reduction in arch depth.

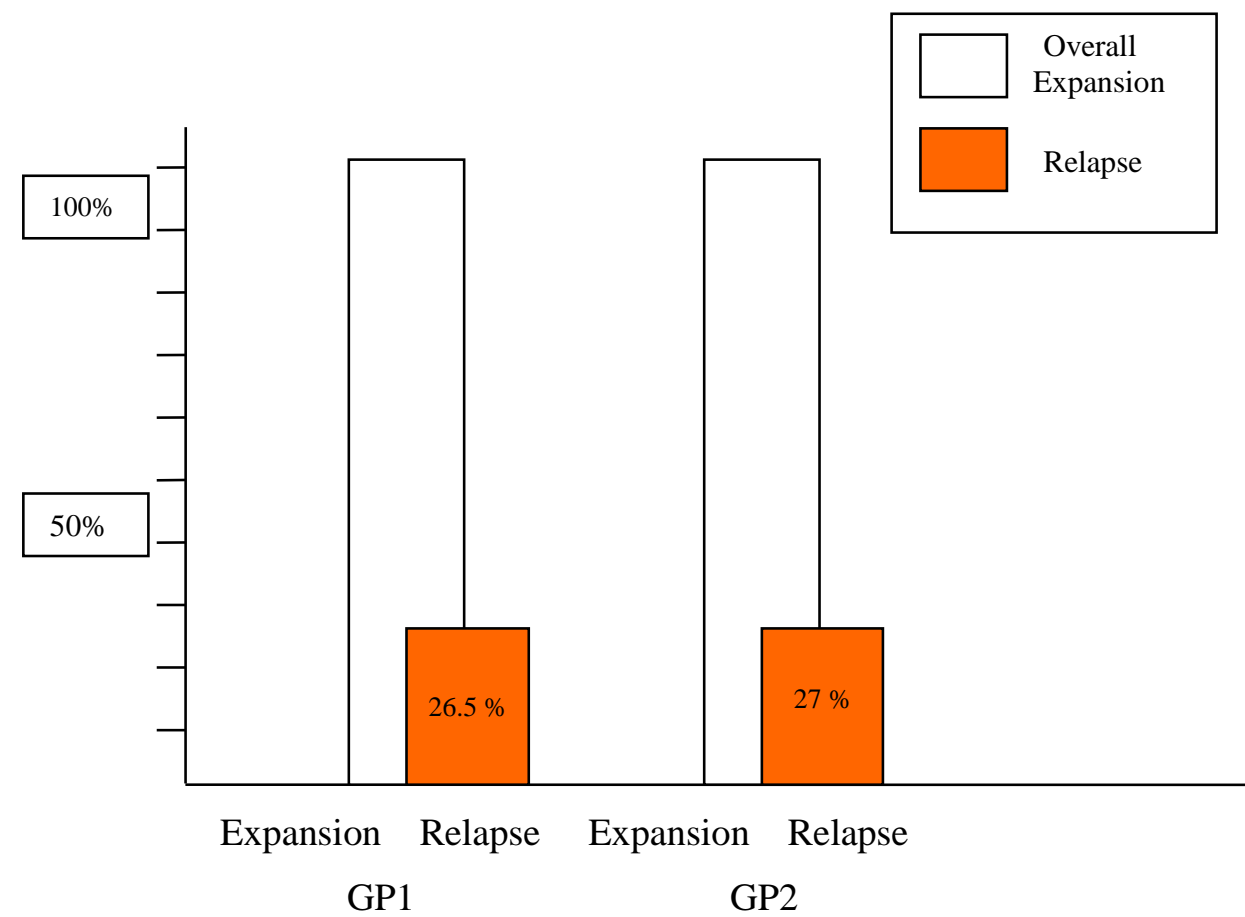

Histogram representing the overall (dental and skeletal) expansion and relapse in Gp1(26.5\%)and Gp2(27\%).

Radiographic Findings

- Occlusal R/Gs at the end of active treatment period showed a radiotransparency along the mid-palatal suture.

- Lateral Cephalograms

Superimposition of the lateral head films taken before and after the expansion and at the end of the observation period, as well as the determination of the angles SNA, ANB, SN-MGo, SN-ANS/PNS and ANS/PNS- Me-Go revealed no significant changes during the experiment in all patients. 
During the expansion period, the SNA angle increased 1 degree(mean), and the ANB angle decreased 0.5 degree during the retention and post-retention phases. The tendency towards an opening of the MPA, and a decrease of the SN-ANS/PNS angle was generally observed during the expansion phase; however non of the values varried more than 2 degrees from the original in the two groups .

These values returned towards their initial readings during the post expansion period.

There was a slight increase in MPA of 1.08 degree, FH (NA-Me) clinically increased very slightly but was statistically significant at $0.25 \pm 0.18$.

No significant changes occurred in PPA, upper AFH (Na-IE) or upper PFH (DCT-SN). No significant changes were found in palatal depth.

\section{DISCUSSION}

In Sagittal Plane: Our results have showed that there was a significant forward displacement of the maxilla ,this finding was in agreement with (Davis and Kronman,1969; and Akkaya,1999).

In vertical Plane: In this study we found that after (RME) treatment, the mandible has rotated downward and backward, which resulted in a smaller SNB angle, higher mandibular plane angle, and longer anterior facial height. Previous studies have also showed an increase in mandibular plane angle (Davis and Kronman,1999;wertz,1970; and Silva Filho etal.,1991).

Other studies have showed a decrease in SNB angle (Silva Filho etal.,1991).

- Our data also showed that the palatal plane has displaced downward after (RME) treatment, similar results have also been reported by Hass(1961),Davis and Kronman(1969),Wretz(1970), and Wretz and Deskin(1977) in children after treatment with (RME).

In addition, other studies have showed that (RME) treatment has increased air flow and improved nasal breathing (Basciftci FA,2002). However this was not investigated in our study. 
Egyptian

Orthodontic Journal

\section{CONCLUSIONS}

* (RME) therapy with banded teeth and 9-11mm expansion create a significant increase in MPA and (NA-Me) FH, but neither increase was great enough to be of clinical importance.

* (RME) appliances were found to be able to produce significant increases

* in intermolar width, palatal width, alveolar tipping and molar tipping.

* (RME) appliances were capable of expanding the maxilla and correction of cross bite.

- BDS.Jordanian National Dental Board(JNDB).Orthodontist at (KHMC).

- BDS.Jordanian National Dental Board(JNDB).Orthodontist at(KHMC).

- BDS,MSc.London.Prosthodontist at(KHMC).

\section{REFERENCES}

1. Akkaya S,Lorenzon S, Üçem TT. A comparison of sagital and vertical effects between bonded rapid and slow maxillary expansion procedure. Eur J Orthod 1999;21:175-80.

2. Basciftci FA, Mutlu N,Karaman AL, Malkoc S, Küçükkolbasi H. Does the timing and method of rapid maxillary expansion have an effect on the changes in the nasal dimension. Angle Orthod 2002;72:118-23.

3. Davis WM, Kronman JH. Anatomical changes induced by splitting the mid palatal suture. Angle Orthod 1969;39:126-32.

4. Grey LP.Rapid maxillary expansion and impaired nasal respiration. Ear Nose Throat J 1987;66:248-51.

5. Hass AJ, 1961, Rapid expansion of the maxillary dental arch and nasal cavity by opening the mid palatal suture. The Angle Orthodontist 47:186-192.

6. Hass AJ. The treatment of maxillary deficiency by opening the mid-palatal suture. Angle Orthod 1965;35:200-17.

7. Isaacson RJ,Wood J L, Ingram AH 1964, forces produced by rapid maxillary expansion.111. Forces present during retention. The Angle Orthodontist 35:178-186.

Volume 27 - June 2005 
8. Silva Filho OG,Villas Boas MC, Capelozza Filho L. Rapid maxillary expansion in the primary and mixed dentitions: a cephalometric evaluation. AM J Orthod Dentofacial Orthop 1991;100:171-81.

9. Wertz RA 1970, Skeletal and dental changes accompanying rapid mid palatal suture opening. American Journal of Orthodontics 58:41-66.

10. Wertz RA, Dreskin M 1977 Mid Palatal suture opening: A normative study. American Journal of Orthodontics 71:367-381. 